\title{
Aux sources de La Rue des Mouches (2005) de Sony Labou Tansi
}

\section{Suzanne Nzouzi}

\section{OpenEdition}

\section{Journals}

Édition électronique

URL : https://journals.openedition.org/coma/7153

DOI : $10.4000 /$ coma. 7153

ISSN : 2275-1742

\section{Éditeur}

Institut des textes \& manuscrits modernes (ITEM)

\section{Référence électronique}

Suzanne Nzouzi, «Aux sources de La Rue des Mouches (2005) de Sony Labou Tansi », Continents manuscrits [En ligne], 17 | 2021, mis en ligne le 15 octobre 2021, consulté le 11 janvier 2023. URL http://journals.openedition.org/coma/7153 ; DOI : https://doi.org/10.4000/coma.7153

Ce document a été généré automatiquement le 11 janvier 2023.

\section{(c) $(1) \&$}

Creative Commons - Attribution - Pas d'Utilisation Commerciale - Pas de Modification 4.0 International - CC BY-NC-ND 4.0

https://creativecommons.org/licenses/by-nc-nd/4.0/ 


\title{
Aux sources de La Rue des Mouches (2005) de Sony Labou Tansi
}

\author{
Suzanne Nzouzi
}

\section{Introduction}

1 Cet article se propose de mettre en lumière la genèse de la pièce de théâtre La Rue des Mouches de Sony Labou Tansi, écrivain francophone africain du $\mathrm{xx}^{\mathrm{e}}$ siècle dont l'œuvre littéraire ${ }^{1}$ prolifique ne cesse d'être renouvelée par les travaux génétiques entrepris par l'équipe Manuscrits francophones au sein de l'Institut des textes et manuscrits modernes (ITEM, CNRS-ENS), laboratoire du CNRS situé à Paris.

Publiée en 2005 aux éditions Théâtrales ${ }^{2}$, cette pièce est une comédie tragique qui met en scène l'opposition entre deux hommes puissants, le seigneur Amalfet et le seigneur Kuti-Kuta. Les habitations de ces deux familles sont séparées par la rue des Mouches. Opposé à la construction de l'usine du seigneur Amalfet dans la rue des Mouches, le seigneur Kuti-Kuta veut faire disparaître ce dernier et se confie à son domestique Essaïne qui va choisir le fou Adiabanko pour commettre cet acte meurtrier en incendiant la bibliothèque du seigneur Amalfet. Adiabanko a été maudit par sa mère après avoir tué ses deux sœurs dans un tour de sorcellerie. Personnage central de la pièce qui entonne également des chants, Adiabanko est à la fois le frère cadet du seigneur Amalfet et un ami des Kuti-Kuta. Il entretient également une liaison amoureuse secrète avec la femme de son frère, Messaïne. Après l'incendie de sa bibliothèque, désespéré, le seigneur Amalfet se donne la mort en se jetant dans l'incendie. Personnage à la fois comique et tragique, Adiabanko tient à la fois des propos incohérents et des paroles très profondes. Regrettant son acte et touché par ce drame, il organise la veillée de son frère dans le respect des traditions. Une ombre, un spectre et des voix apparaissent et sont entendus dans la rue des Mouches, terrorisant les habitants qui quittent les lieux, sauf le seigneur Kuti, son épouse et son domestique qui restent sur les lieux. Devenu dépressif, le seigneur Kuti devient dépendant aux antidépresseurs. Déguisé en percepteur d'impôts, Adiabanko tue le domestique des 
Kuti-Kuta, Essaïne, d'un coup de poignard, et lui-même est tué à la fin de la pièce par un coup de fusil du seigneur Kuti-Kuta. Mais avant sa mort, il guérit de sa folie.

Cette étude analyse les différentes étapes de la constitution du texte et met en évidence le chantier de La Rue des Mouches, pièce très inspirée des traditions congolaises, puis se penche sur la datation du texte et la mise en scène, fruit d'une collaboration francocongolaise. Enfin, elle rend compte de la réception de cette pièce dans les milieux du théâtre francophone.

\section{Dossier génétique}

\section{Manuscrit}

4 C'est un manuscrit autographe provenant du fonds Brazzaville, et déposé à la Bibliothèque francophone multimédia de Limoges. Il se présente sous la forme d'un cahier de grand format $(29 \times 21 \mathrm{~cm})$, comprenant 24 folios (soit 59 pages dont 11 blanches). Il a été inventorié en 2008 sous le numéro 11 (cf. Études littéraires africaines, $\left.\mathrm{n}^{\circ} 15\right)$ et rangé sous la cote : RES.PF SLT 7.

Le cahier est abîmé. Sa couverture est bleue, ses bordures sont déchirées par endroits. La $4^{\mathrm{e}}$ de couverture et la dernière page sont déchirées en haut à gauche. Il comprend plusieurs textes et des notes variées :

- un texte narratif titré Clair de lune à Rocheau pour Janvio Saravana en 4 feuillets ;

- un texte intitulé Sire Katawa bra Kuti en 12 feuillets ;

- un texte intitulé La semaine de huit jours en 52 feuillets ;

- un ensemble de 10 feuillets ayant pour titre Projet d'aménagement d'une coopérative communale : Théâtre Rocado Zulu.

6 Le premier texte s'apparente à une nouvelle, le second et le troisième sont des pièces de théâtre, le quatrième est une lettre.

7 Les deux ébauches de La Rue des Mouches, à savoir Sire Katawa bra Kuti et La semaine de Huit jours, n'ont pas la même configuration.

1. La première ébauche comprend des notes variées portant sur la littérature, une liste de 11 personnages, des notes sur une page titrée « Littérature et théâtre » et enfin, une scène courte suivie de notes diverses. Cette version met l'accent sur un cadeau adressé à sire Kuti qui a organisé une fête dans sa villa, mais ses proches ne veulent pas le lui remettre car il en mourrait de chagrin. Son épouse, Lady K ou Elisabeth, qui deviendra Laoudia dans les tapuscrits, est déçue car son mari a été bon envers les gens qui le lui rendent par le mépris et la haine.

2. La deuxième ébauche est une suite de textes inachevés intitulés : "Scène 1 ", «Scène 1 La veillée », un « Prologue » en vers, encore « Scène 1 », puis « Scène 2 ». Dans cette version, tous ceux qui habitaient chez sire Kuti-Kuta s'en vont apeurés par le spectre, l'ombre et la voix qui serait celle du fantôme du seigneur Amalfet dans la rue des Mouches. Propriétaire terrien puissant, sire Kuti se sent trahi ; il devient méfiant et hystérique, il souffre de cette tension. Il est, par ailleurs, en conflit avec sire Amalfet, militaire qui porte le titre de général, car ce dernier a relancé son chantier de construction de l'hôtel Gariama International dans la zone protégée des Kuti-Kuta, chantier qui avait été annulé grâce à l'influence du seigneur Kuti, mais neuf ans après, le seigneur Amalfet a obtenu le droit de construire. Les deux camps arborent des 
couleurs différentes qui marquent leur opposition et leurs maîtres respectifs se disputent et s'insultent. Ceux qui ont quitté le seigneur Kuti, qui a perdu ses richesses et son honneur, sont désormais au service du seigneur Amalfet qui s'en vante auprès de son ennemi. Dans une hallucination, le seigneur Kuti voit son ennemi entrer chez lui pour lui annoncer qu'il lui enverra son orchestre afin de lui chanter sa ruine; il chante alors le refrain de la Merdesa. Ce chant est repris par un étranger, le percepteur, un colosse qui vient annoncer la mort des deux seigneurs quatre ans auparavant: celle d'Amalfet dans un accident de chasse à Rochoto et celle de Kuti-Kuta dans une noyade dans le lac Koyo. Puis, le percepteur sort un registre des impôts qu'il consulte. Ainsi, s'achève le texte.

\section{Tapuscrits}

Tps 1 : 28 pages (fonds Philippe Sita ${ }^{3}$, Brazzaville)

9 Tps $2: 42$ pages reliées par une spirale (fonds Philippe Sita, Brazzaville)

Tps $3: 47$ pages (BFM, Limoges)

11 La numérotation des tapuscrits correspond ici à l'ordre chronologique dans lequel nous les présentons.

Tps 1

12 C'est un document qui comporte 28 pages; il est composé de deux ensembles hétérogènes : 12 pages recto (le texte de la pièce) et les autres pages verso, utilisées pour des notes diverses dont certaines ont un lien avec la pièce.

13 Ce sont des notes manuscrites autographes et allographes, dont le rapport n'est pas toujours évident à établir avec le manuscrit et la version imprimée, qui portent à la fois sur le mysticisme (les fantômes, le fétichisme, l'occultisme, la magie et la religion), sur la vie politique congolaise vraisemblablement du début des années 90 et surtout syndicale, et enfin sur les maladies et notamment le mwanza ${ }^{4}$. Les notes sur les fantômes permettent de comprendre la présence de l'ombre qui habite La rue des Mouches. Signalons également la présence d'une page dactylographiée (page 23) qui correspond vraisemblablement à un avant-texte de Monologue d'or et noces d'argent ${ }^{5}$, dont les caractères sont de couleur plus claire et plus petits que le reste du tapuscrit.

14 Ce tapuscrit est visiblement lacunaire : des pages et des scènes sont manquantes, de nombreuses scènes sont incomplètes par rapport à la version imprimée. Il est annoté et quelques notes sont illisibles. Il ne comporte pas de page de titre, mais une première page où figurent un avertissement et la liste des personnages. Ce document ne comporte quasiment pas de ratures.

Les modifications du texte - qui sont surtout des ajouts - sont portées à l'encre bleue et noire et concernent surtout la mise en scène. De nombreuses notes sont en français et en anglais, ce qui constitue un cas unique dans le corpus sur lequel nous avons travaillét. Rappelons que Sony avait été professeur d'anglais au collège de 1971 à 1979 dans les localités provinciales du sud du Congo. 
Tps 2 effacée par des taches; elle mentionne les personnes présentes et leur heure d'arrivée, ainsi que les absents. Dans la marge droite, tout en haut de la page, est mentionnée la date « 5-85».

21 Le texte des tapuscrits étant plus travaillé et plus étoffé que le texte de la version manuscrite, on peut affirmer que ce sont des versions postérieures à la version manuscrite. On peut également penser que les notes allographes de mise en scène dans les deux premiers tapuscrits sont intervenues tardivement, notamment après 1987, si l'on s'en tient au fait que Philippe Sita a rejoint la troupe tardivement en 1987 où il a joué le rôle de Jullius Caïd Kaesaire dans Moi, Veuve de l'Empire (1987). Rappelons, par ailleurs, que La Rue des Mouches (2005) a été créée entre autres à Limoges en octobre 1985, à Paris en novembre 1985 et à Brazzaville en janvier 1986, donc avant l'arrivée de Philippe Sita dans la troupe, puis en juin 1995 à Brazzaville, après la mort de Sony. 

l'hypothèse selon laquelle Sony aurait confié les deux tapuscrits à Philippe Sita pour une relecture, une correction, avec un travail sur la mise en scène et une mise en forme de la musique avant l'envoi chez l'éditeur, sachant que c'est un texte posthume qui a été publié en 2005 par Bernard Magnier aux Éditions Théâtrales, à Montreuil. La grande majorité de ces notes portent sur la mise en scène et certaines sont des chants kongos qu'on entend dans la représentation de la pièce à Limoges. Quelques notes n'ont cependant pas été maintenues dans la version imprimée.

deux personnages qui apparaissent dans une nouvelle et un roman de Sony rédigés dans les années 80. Il s'agit, tout d'abord, du personnage principal de L'image ${ }^{9}$ (1986), Jean-Max Sadoun, un Blanc influent et riche qui a été rebelle pendant quarante-sept ans et qui, à la fin de la nouvelle, est menacé d'une arme à feu chez lui par un homme nommé Petillo Argentillo do Mboudo Nisa, lequel l'accuse d'avoir menacé de mort un certain Manuello de Conzardo Santos. Or, le nom de l'agresseur de Jean-Max Sadoun, Petillo Argentillo do Mboudo Nisa, a été emprunté au nom d'un ami de Sony, Alphonse Mboudo-Nesa, qui a été un mécène de la troupe du Rocado Zulu Théâtre, ancien ministre du Commerce et directeur adjoint d'AGIP ${ }^{10}$, et ancien ministre des Mines et de l'Énergie dans le gouvernement du commandant Louis-Sylvain Ngoma sous la présidence de Marien Ngouabi en 1975. Le deuxième personnage est Diégo Sadoun Argandov dans Les Yeux du volcan ${ }^{11}$ (1988), l'aîné de la puissante famille Argandov et personnage influent auquel le héros du roman, un colosse, refuse de vendre des crimes contre de l'argent. D'ailleurs, on peut faire un rapprochement entre le colosse du roman et celui qui apparaît à la fin du premier manuscrit dans le texte La Semaine de huit jours.

Les thèmes du pouvoir et de la mort qui traversent L'image et Les Yeux du volcan sont également très présents dans $\mathrm{L} a$ Rue des Mouches, où l'intrigue repose sur le spectre du seigneur Amalfet qui apparaît de manière récurrente dans la rue des Mouches, après son suicide provoqué par le seigneur Kuti-Kuta qui n'a pas supporté qu'il construise son usine à côté de son domicile.

\section{Création}

La Rue des Mouches a été créée, en première mondiale, à Eymoutiers (Haute-Vienne), dans le cadre du $2^{e}$ Festival de la francophonie en Limousin ${ }^{12}$ qui s'est tenu du 11 octobre au 25 octobre 1985. Le Rocado Zulu Théâtre s'est rendu en tournée à Limoges et dans six autres villes ${ }^{13}$, puis à Arnac-Pompadour en Corrèze. Première pièce jouée par Sony et sa troupe au Festival de Limoges, elle a ensuite été jouée à Bordeaux, puis à

Continents manuscrits, $17 \mid 202$ 
Paris, précisément à l'Unesco devant «trois mille personnes ${ }^{14}$ » le 7 novembre 1985. Elle a également été présentée au Centre culturel français de Brazzaville le 14 décembre $1985^{15}$, puis a été jouée au même endroit les 16 et 17 juin 1995 après le décès de Sony.

Elle a fait l'objet de plusieurs enregistrements :

- France 3 Limousin-Poitou-Charentes ${ }^{16}$ (sous la direction de Philippe Laïk) ;

- France Culture ${ }^{17}$;

- Institut national de l'audiovisuel.

Mise en scène : Sony Labou Tansi et Pierre Vial.

Assistant : Pascal Nzonzi.

Décor : Émile Mokoko.

Costumes : Motse Akanati et J.-M. Hemilembolo.

Régie : Victor Mbila Mpassi.

Avec : Édith F. Baghamboula / Julienne Milandou (Laoudia), Simone Bakoua / Marie-Léontine Tsibinda (Messaïne), Nicolas Bissi (Essaïne), Georges M’Boussi (Amalfet), Jonas Dian Daha (première voix), Éric Mampouya (seconde voix), Michel Matondo (Ossombro), Joseph Missakidi (Adiabanko), Victor Mpene Malela Louya (Kuti-Kuta), le Chœur (Édith F. Baghamboula, Simone Bakoua, Georges M’Boussi, Jonas Dian Daha, Éric Mampouya, Michel Matondo, Julienne Milandou).

L'arrivée de Pierre Vial à Brazzaville en 1985, et précisément au mois de juillet ${ }^{18}$ en pleine saison sèche, pour les répétitions d'une durée d'un mois avec Sony et le Rocado Zulu Théâtre est mentionnée par Victor Mbila-Mpassi dans l'entretien réalisé en 2019 avec Julie Peghini, Nicolas Martin-Granel et Sonia Lemoigne-Euzenot ${ }^{19}$.

D'après Victor Mbila-Mpassi, l'acteur congolais Pascal Nzonzi, qui était l'assistant de Pierre Vial, aurait servi d'intermédiaire entre Pierre Vial et Sony. Il a été, de surcroît, assistant de Pierre Vial et de Sony dans la mise en scène de La Rue des Mouches.

Témoin de la collaboration entre les deux metteurs en scène, Victor Mbila-Passi souligne le fait qu'un grand travail avait été effectué par Sony et ses comédiens avant l'arrivée de Pierre Vial. En effet, le Rocado Zulu Théâtre avait pour habitude de multiplier les répétitions à l'approche du Festival de Limoges pour laquelle la troupe montait des pièces chaque année de 1985 à 1989, comme l'affirme ici le régisseur de la troupe : «Par exemple, pour La Rue des Mouches qu'on devait présenter en septembre, tout le mois d'août on travaille de 8 heures à 22 heures..$^{20}$ "

D'après lui, Pierre Vial avait un regard différent de celui de Sony ; c'est lui qui dirige les répétitions à son arrivée, il reprend la pièce " scène par scène ${ }^{21}$ » et Sony va s'effacer. Quant à Victor Mbila-Mpassi, il prend des notes. Ce dernier a beaucoup appris du metteur en scène français qu'il trouvait, par ailleurs, très modeste et décrit ici cette collaboration : «Les lumières, je m'en souviens, la première esquisse du plan de feuille, on l'a fait ensemble. Il m'a expliqué : "telle scène, c'est la peur". "L'autre scène, plus énergique". ${ }^{22}$ " Il lui a, d'ailleurs, proposé une formation qu'il fera auprès de Pierre Debauche, à l'époque directeur du Centre dramatique national de Rennes ${ }^{23}$. 


\section{Réception}

D'après Pierre Debauche, cité par Caya Makhélé ${ }^{4}$ dans la revue parisienne Notre librairie en 1993, La Rue des Mouches a fait entrer le conte et la musique dans le Festival de Limoges, permettant ainsi la découverte de la culture congolaise et la rencontre avec l'autre. D'ailleurs, ces échanges et ces rencontres multiples à travers le théâtre ont conduit au changement du nom de ce festival qui devient en 1987, soit à sa quatrième édition, le Festival international des francophonies.

Parmi les différentes troupes de théâtre ayant joué à Eymoutiers, le Rocado Zulu Théâtre s'est démarqué par la richesse et l'authenticité de son travail, soulignées par un journaliste de Libération le 31 octobre 1985, dont les propos ont été cités dans La Semaine africaine en décembre 1985 par Marie-Léontine Tsibinda, comédienne congolaise de la troupe qui interprète en partage avec Simone Bakoua le rôle de Messaïne dans la pièce, l'épouse du seigneur Amalfet : «[...] le travail du Rocado appartient à un univers spécifique qui ne relève plus simplement d'une pratique héritée de l'Occident (le théâtre), à la tradition (le rituel mêlé à la musique et la danse...) ils en furent (les spectacles) pourtant de loin les plus riches et les plus novateurs. ${ }^{25}$ " En effet, la pièce développe des thèmes variés parmi lesquels les traditions congolaises, les différences, la séparation entre les deux Congos et la séparation entre l'Europe et l'Afrique comme le souligne Sony dans l'introduction de la pièce ${ }^{26}$. Dans ce même article, Marie-Léontine Tsibinda, qui quittera la troupe après la représentation d'Antoine m'a vendu son destin ${ }^{27}$ à Limoges en 1986, souligne que «les acteurs de la troupe devant ce public nouveau [...] ont donné le meilleur d'eux-mêmes, car à travers leur jeu, c'est la culture d'un pays que l'on mettait sur la sellette ${ }^{28}$ ».

Fig. 1 : Les comédiens du Rocado Zulu Théâtre au Festival de la francophonie

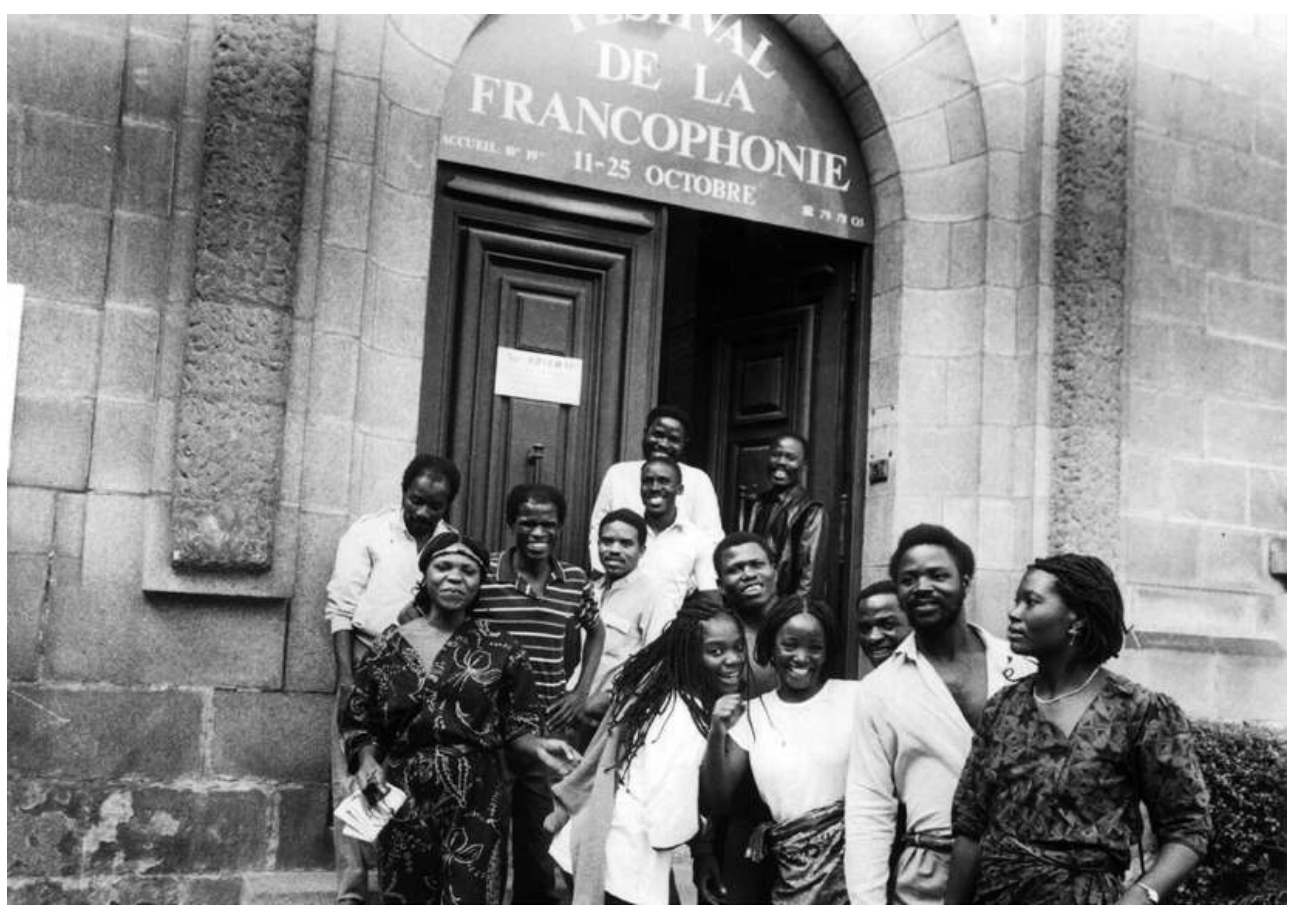

(c) Les Francophonies en Limousin, 1985 

est intéressant de constater qu'il n'apparaît à proprement parler pas dans le manuscrit, où le texte est à l'état de chantier et se construit, à moins de considérer que le percepteur qui apparaît dans la scène 2 à la fin du cahier et qui évoque la mort des seigneurs Amalfet et Kuti est en réalité le fou Adiabanko, car dans les tapuscrits 2 et 3 ce dernier se déguise en percepteur pour pénétrer chez le seigneur Kuti-Kuta et le tuer. Africains n'ont pas attendu l'arrivée des Occidentaux en Afrique pour faire du théâtre, car cette pratique existait déjà sous des formes traditionnelles intégrées dans la vie sociale, comme il l'affirmait ici en 1988: «Là-bas, avant l'arrivée des Européens, nous avions cinq formes de théâtre qui présentaient toutes un lien entre la vie quotidienne et la vie artistique ${ }^{29}$.» En effet, la tradition congolaise renferme beaucoup de théâtralité.

Dans l'Avertissement de la pièce, Sony lui-même situe sa pièce dans un cadre bien précis, celui d'une tradition ancestrale congolaise :

Cette pièce s'inscrit dans le cadre d'un thêâtre vieux comme le monde kongo, le kinguizila ou théâtre de la guérison. Il consiste à donner un rôle à jouer dans une histoire créée à un malade, dans l'unique but de le guérir. L'histoire peut connaître quelques improvisations. Ici, le malade à guérir est le fou Adiabanko, cadet du seigneur Amalfet, ami des Kuta. Je prends l'histoire à la neuvième séance. Les actes posés sont vrais, ainsi que le texte conçu ${ }^{30}$.

Dans une série d'entretiens réalisés en décembre 2017 à Brazzaville par Julie Peghini et Nicolas Martin-Granel, Matondo Kubu Turé, un homme de théâtre congolais très proche de Sony, qui dirigeait la troupe artistique Ngunga à Brazzaville dans les années 80 et qui a joué dans des pièces de théâtre de Sony, soulignait la difficulté de mettre en scène certains rites traditionnels, dont le kinguizila qui «[...] est essentiellement basé sur de la transe, des cris, il n'y a pas de texte, des danses. C'est un fétiche, une sorte de cérémonie rituelle d'une société secrète. Un rituel sans masques... $31 \gg$.

Ce faisant, ainsi que le souligne Georges M'Boussi, l'un des anciens comédiens du Rocado Zulu Théâtre ayant joué le rôle du seigneur Amalfet dans la pièce, Sony « [...] réactualise une tradition et influence une pratique théâtrale moderne ${ }^{32}$. " Georges $\mathrm{M}$ 'Boussi va un peu plus loin, sans pour autant nommer les dangers liés à la pratique de ce rite fétichiste. Il évoque seulement « [...] tous les risques qu'il comporte, par rapport à ses débordements ${ }^{33}$ ».

Dans notre entretien téléphonique ${ }^{34}$ du 8 juin 2020, Édith Bagamboula, ancienne comédienne du Rocado Zulu Théâtre ayant joué le rôle de Laoudia, l'épouse du seigneur Kuti-Kuta, nous a informé que certains comédiens étaient entrés en transe en pleine représentation de L'arc-en-terre pendant qu'ils interprétaient des chants et des rites du kinguizila, pièce jouée au deuxième Festival de Limoges en 1985.

Georges M'Boussi cite, d'ailleurs, trois comédiens de Sony versés dans ce rite, à savoir «[...] Faustin Mouanga Sengha, comédien, poète, détenteur des rites traditionnels, [...] Louya Victor Mpene Malela qui dans son enfance avait vécu le rite kinguizila dans son village Yanga Nzala, et d'autres artistes ${ }^{35}$ ».

Concernant le personnage énigmatique du fou à guérir, en l'occurrence Adiabanko, il
ce dernier se déguise en percepteur pour pénétrer chez le seigneur Kuti-Kuta et le tuer. 

l'écrivain, comme le soulignait Tchicaya U'Tamsi en 1985, en rappelant le caractère jadis sacré de la personne du fou et en voyant en Dadou, personnage principal du roman L'Anté-Peuple (1985), un double de Sony : « Sony est fou sacré qui fait l'éloge de la folie agissante. Il est un fou-oracle qui dit des augures ${ }^{36}$. » D'ailleurs, Sony a dédidacé $L a$ Rue des Mouches aux fous : «À tous les fous de La Peau cassée / À tous ceux qui voient venir un autre monde ${ }^{37}$ ". L'attachement de Sony au thème de la folie se confirme avec la présence dans sa bibliothèque personnelle des Contes de la folie ordinaire de Charles Bukowski (en livre de poche), bibliothèque dont l'inventaire a été effectué par Nicolas Martin-Granel ${ }^{38}$ à Brazzaville en 1997. Cet inventaire nous permet, de plus, d'affirmer que Sony lisait beaucoup et avait lu Jean-Paul Sartre ${ }^{39}$, lequel l'a sans doute influencé, si l'on s'en tient aux nombreuses analogies entre La Rue des Mouches et Les Mouches (1947), notamment à travers les thématiques de la mort et du deuil, du complot et de la vengeance, du travestissement et de l'amour, même si la pièce de Sartre reprend le mythe grec tragique des Atrides sur un ton léger. On trouve également des points communs entre la pièce de Sony et Sa majesté des Mouches (1956) du britannique William Golding, notamment à travers les thèmes du pouvoir et de la loi.

Outre la présence des rites traditionnels dans la pièce, la place occupée par la musique, et particulièrement la musique congolaise, est prépondérante, comme le soulignait Mukala Kadima-Nzuji en 1993 dans la revue Notre librairie ${ }^{40}$, après avoir relevé que le titre même de la pièce renvoyait à une célèbre chanson de la variété congolaise (RDC), «Balabala», mot lingala qui signifie «rue» en français. Cette chanson de la fin des années 60 est une création du docteur Nico et de l'orchestre African Fiesta, dont le titre officiel est « Kiri-kiri mabina ya sika ${ }^{41}$ ». C'est l'histoire d'un homme amoureux d'une femme nommée Angèle qu'il cherche dans les rues de Kinshasa, mais qui a déménagé et qu'il veut retrouver.

Enfin, Sony lui-même a commenté dans la presse congolaise en 1986 le décor qu'il avait choisi pour cette mise en scène qui laisse voir un long tissu étalé sur le sol à la fois blanc et marron, voire rouge bordeaux, qui incarne la rue des Mouches et à l'extrémité gauche de la rue, le visage en gros plan d'une sorte de statue très colorée qui comporte de gros traits, dont la forme et les couleurs vives évoquent les mondes africain et amérindien $^{42}$ : «La peinture congolaise était honorée, à cause du décor d'Émile Mokoko ${ }^{43}$.» Quant à Victor Mbila-Mpassi, il ajoute: "Une toile qui arrivait dans la scène, avec des pas. Le peintre exécute en apportant aussi ces idées ${ }^{44}$. » 
Fig. 2 : Décor pour La Rue des mouches de Sony Labou Tansi, par le Rocado Zulu Théâtre

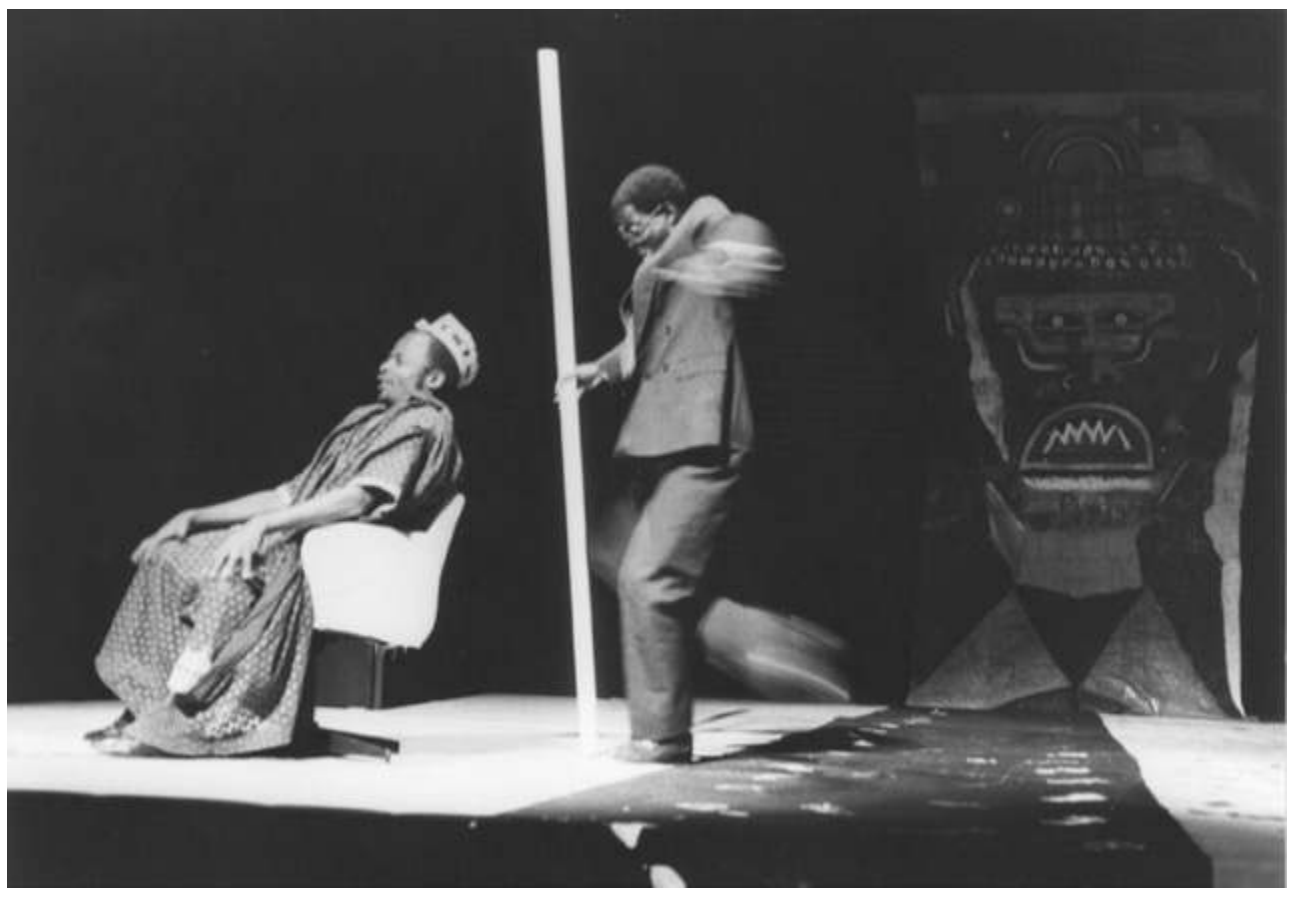

(c) Jean Goussebaire, Les Francophonies en Limousin, 1985

\section{NOTES}

1. Si l'on tient seulement compte des œuvres publiées, Sony Labou Tansi a rédigé une vingtaine de pièces de théâtre, une dizaine de nouvelles ainsi que six romans, en plus des quatre recueils de poésie, des nombreux poèmes épars publiés dans des revues et dans des ouvrages collectifs.

2. Sony Labou Tansi, «La Rue des Mouches », pp. 13-42 dans Bernard Magnier, Sony Labou Tansi. Paroles inédites, Éditions théâtrales, Paris, 2005, 127 p.

3. Il est à la fois auteur, compositeur, interprète, guitariste et conteur. Il a été comédien et musicien du Rocado Zulu Théâtre de 1987 à 1990.

4. Maladie qui affecte la peau.

5. Sony Labou Tansi, Théâtre 3. Monologue d'or et noces d'argent suivi de Le Trou, Belgique, Lansman, $1998,91 \mathrm{p}$.

6. Ce corpus est constitué de La Rue des Mouches (2005), La Résurrection rouge et blanche de Roméo et Juliette (1990) et Moi, Veuve de l'Empire (1987).

7. Metteur en scène et professeur au Conservatoire national d'art dramatique de Paris de 1975 à 1993.

8. Entretien du 24 décembre 2019 réalisé à Brazzaville par Julie Peghini, Nicolas Martin-Granel et Sonia Lemoigne-Euzenot (p. 4).

9. « L'image », Revue Équateur (Paris), n 1, octobre-novembre 1986, p. 55-58.

10. Compagnie pétrolière italienne qui possède une filiale à Pointe-Noire, capitale économique de la République du Congo. 
11. Sony Labou Tansi, Les Yeux du volcan, Paris, Seuil, 1988, 191 p. Ce personnage apparaît aux pages 23 et 53 du roman.

12. L'acteur et metteur en scène belge Frédéric Dussenne a fait une lecture de la pièce lors des $22^{\text {es }}$ Francophonies en Limousin, dans le cadre des «Écrits de résidents ".

13. Ces villes sont Aixe-sur-Vienne, Eymoutiers, Bessines-sur-Gatempes, Magnac-Laval, SaintJunien et Saint-Yrieix.

14. Apollinaire Singou-Basseha, «Le "prix AGIP-recherches" à Sony Labou Tansi », La Semaine africaine (Brazzaville), 3-8 janvier 1986, p. 9.

15. Marie-Léontine Tsibinda, « Rocado Zulu Théâtre. Le Festival de la Francophonie ou la soif de l'autre », La Semaine africaine (Brazzaville), 23 décembre 1985-2 janvier 1986, p. 8.

16. L'enregistrement dure $1 \mathrm{~h} 01 \mathrm{mn} 26 \mathrm{~s}$ et ne contient pas la totalité de la pièce, mais uniquement le premier tableau qui renferme trois scènes.

17. Nous avons adressé un courriel à France Culture pour accéder à cette archive, mais France Culture nous a renvoyé vers l'INA. Cependant, une présentation de la pièce est accessible au lien suivant : https://vimeo.com/133658532

18. Caroline Bourgine, "Francophonies dans un magasin de porcelaines ", Libération, mardi 6 octobre 1987, p. 42.

19. Entretien du 24 décembre 2019 réalisé à Brazzaville par Julie Peghini, Nicolas Martin-Granel et Sonia Lemoigne-Euzenot (p. 1).

20. Ibid., p. 2. Nous n'avons aucune information sur le lieu où a été jouée la pièce en septembre.

21. Ibid., p. 3.

22. Ibid., p. 4.

23. Ibid., p. 1.

24. Caya Makhélé, «Présence et contradictions du théâtre africain », p. 21 dans Créateurs africains à Limoges, Paris, Clef, coll. « Notre librairie », nº Hors-série, septembre 1993.

https://gallica.bnf.fr/ark:/12148/bpt6k6495063q/f22.item

25. Marie-Léontine Tsibinda, « Rocado Zulu Théâtre. Le Festival de la Francophonie ou la soif de l'autre ", art. cit., p. 8.

26. Sony Labou Tansi, «La Rue des Mouches", p. 15 dans Bernard Magnier, Sony Labou Tansi. Paroles inédites, Éditions théâtrales, Paris, 2005, 127 p.

27. Sony Labou Tansi, Antoine m'a vendu son destin, Paris, Acoria, 1997, 63 p.

28. Marie-Léontine Tsibinda, "Rocado Zulu Théâtre. Le Festival de la Francophonie ou la soif de l'autre », art. cit., p. 8.

29. Assiatou Diallo Bah, "Sony Labou Tansi ", Amina (Paris), n²20, août 1988, p. 55. Il existe plusieurs formes de théâtre kongo parmi lesquels : le kinguizila (le théâtre de guérison), le nkoloba (le théâtre des marionnettes), le lemba (le théâtre d'initiation à la richesse), le saambou (le théâtre de la contagion), le yala-yala ou nsimba (le théâtre des jumeaux) et l'insulte publique (le théâtre des rois). Dans le théâtre kongo, le corps, la musique, la danse, l'improvisation et l'implication du public occupaient une place considérable.

30. Sony Labou Tansi, « La Rue des Mouches », dans Bernard Magnier, op. cit., p. 14.

31. Entretiens réalisés en décembre 2017 par Julie Peghini et Nicolas Martin-Granel avec Matondo Kubu Turé, p. 8.

32. Nicolas Martin-Granel, Julie Peghini, La Chair et l'Idée, Besançon, Les Solitaires intempestifs, 2015, p. 186.

33. Nicolas Martin-Granel, Julie Peghini, La Chair et l'Idée, op. cit., p. 187.

34. Entretien téléphonique du 8 juin 2020 avec Édith Bagamboula.

35. Nicolas Martin-Granel, Julie Peghini, La Chair et l'Idée, op. cit., p. 187.

36. Tchicaya U’Tamsi, « Sony Labou Tansi le voyant », Libération (Paris), 7 mars 1985, p. 28.

37. Sony Labou Tansi, « La Rue des Mouches », dans Bernard Magnier, op. cit., p. 13. 
38. Nicolas Martin-Granel, «Inventaire de la bibliothèque de Sony Labou Tansi », p. 41 dans Études littéraires africaines (Paris), $\mathrm{n}^{\circ}$ 15, mai 2003, p. 39-46.

39. Jean-Paul Sartre, Le Diable et le Bon Dieu, Folio (annoté par Sony), dans Nicolas Martin-Granel, "Inventaire de la bibliothèque de Sony Labou Tansi », art. cit., p. 41.

40. Mukala Kadima-Nzuji, «Paroles et musique : pérennité du lien », Notre librairie (Paris), $n^{\circ} 154$, avril-juin 2004, p. 19.

41. Ce titre est en lingala et signifie « Kiri-Kiri, la nouvelle danse ».

42. Annexe $n^{\circ} 13$ : Décor de la scène (p. 18).

43. Apollinaire Singou-Basseha, "Le "prix AGIP-recherches" à Sony Labou Tansi ", La Semaine africaine (Brazzaville), 3-8 janvier 1986, p. 9.

44. Entretien réalisé le 24 décembre 2019 à Brazzaville par Julie Peghini, Nicolas Martin-Granel et Sonia Lemoigne-Euzenot avec Victor Mbila-Mpassi, p. 2.

\section{RÉSUMÉS}

Cet article se propose de mettre en lumière la genèse de la pièce de théâtre La Rue des Mouches de Sony Labou Tansi. L'étude s'attache aux différentes étapes de la constitution du texte et met en évidence le chantier de La Rue des Mouches, pièce très inspirée des traditions congolaises, puis se penche sur la datation du texte et la mise en scène, fruit d'une collaboration franco-congolaise. Enfin, elle rend compte de la réception de cette pièce dans les milieux du théâtre francophone.

\section{INDEX}

Mots-clés : Sony Labou Tansi, théâtre traditionnel congolais, Rocado Zulu Théâtre, Festival des francophonies de Limoges, mise en scène, collaboration franco-congolaise, La Rue des Mouches

\section{AUTEUR}

\section{SUZANNE NZOUZI}

Docteure en littérature et civilisation françaises (Sorbonne Université - Paris IV) et professeure certifiée de lettres modernes au collège (Académie de Créteil), Suzanne Nzouzi a soutenu une thèse en juin 2021 intitulée « Un écrivain populaire africain au XXe siècle : Sony Labou Tansi » sous la direction du professeur Romuald Fonkoua. Elle a collaboré au volume Sony Labou Tansi. Poèmes, paru en 2015 chez CNRS Éditions et collabore actuellement au volume Théâtre. 